\title{
Low BRMS1 expression promotes nasopharyngeal carcinoma metastasis in vitro and in vivo and is associated with poor patient survival
}

Rui-Xue Cui ${ }^{1}{ }^{2+}$, Na Liu ${ }^{1,2+}$, Qing-Mei He ${ }^{2}$, Wen-Fei Li ${ }^{1}$, Bi-Jun Huang ${ }^{2}$, Ying Sun ${ }^{1}$, Ling-Long Tang ${ }^{1}$, Mo Chen ${ }^{1}$, Ning Jiang ${ }^{1,2}$, Lei Chen ${ }^{1}$, Jing-Ping Yun ${ }^{2,3}$, Jing Zeng ${ }^{2,3}$, Ying Guo ${ }^{4}$, Hui-Yun Wang ${ }^{2^{*}}$ and Jun Ma ${ }^{1,2,5^{*}}$

\begin{abstract}
Background: Breast cancer metastasis suppressor 1 (BRMS1) is a metastasis suppressor gene. This study aimed to investigate the impact of BRMS1 on metastasis in nasopharyngeal carcinoma (NPC) and to evaluate the prognostic significance of BRMS1 in NPC patients.

Methods: BRMS1 expression was examined in NPC cell lines using quantitative reverse transcription-polymerase chain reaction and Western blotting. NPC cells stably expressing BRMS1 were used to perform wound healing and invasion assays in vitro and a murine xenograft assay in vivo. Immunohistochemical staining was performed in 274 paraffin-embedded NPC specimens divided into a training set $(n=120)$ and a testing set $(n=154)$.

Results: BRMS1 expression was down-regulated in NPC cell lines. Overexpression of BRMS1 significantly reversed the metastatic phenotype of NPC cells in vitro and in vivo. Importantly, low BRMS1 expression was associated with poor distant metastasis-free survival (DMFS, $P<0.001)$ and poor overall survival $(O S, P<0.001)$ in the training set; these results were validated in the testing set and overall patient population. Cox regression analysis demonstrated that low BRMS1 expression was an independent prognostic factor for DMFS and OS in NPC.
\end{abstract}

Conclusions: Low expression of the metastasis suppressor BRMS1 may be an independent prognostic factor for poor prognosis in NPC patients.

Keyword: BRMS1, Nasopharyngeal carcinoma, Metastasis, Prognosis

\section{Background}

According to the Global Cancer Statistics released by the International Agency for Research on Cancer (IARC), there were an estimated 84,400 incident cases of nasopharyngeal carcinoma (NPC) and 51,600 NPCrelated deaths in 2008 [1]. Highly metastatic NPC is one of the most common malignant tumors in southern China, especially in the province of Guangdong, [2]. For patients with locally advanced disease receiving chemoradiation therapy, the rate of distant metastasis may

\footnotetext{
*Correspondence: wanghyun@mail.sysu.edu.cn; majun2@mail.sysu.edu.cn ${ }^{\dagger}$ Equal contributors

'Department of Radiation Oncology, Sun Yat-sen University Cancer Center, Guangzhou, People's Republic of China

${ }^{2}$ Present address: State Key Laboratory of Oncology in South China, Sun Yatsen University Cancer Center, 651 Dongfeng Road East, Guangzhou 510060, Peoples Republic of China

Full list of author information is available at the end of the article
}

range between 13 and 21\% [3,4]. Studies by our group and others have shown that although the increasingly prevalent use of intensity-modulated radiation therapy (IMRT) and concurrent chemoradiation therapy for locoregionally advanced NPC has improved local and regional control in NPC, distant metastasis has become a predominant pattern of treatment failure in NPC patients who do not present metastases at diagnosis $[5,6]$.

Metastasis is a multistep process in which cancer cells disseminate and establish secondary tumors at sites distant from the primary tumor; metastasis is estimated to be responsible for approximately $90 \%$ of all cancer deaths [7]. The breast cancer metastasis suppressor 1 (BRMS1) gene was originally identified as a true metastasis suppressor gene in breast cancer cell lines as stable overexpression of BRMS1 suppressed pulmonary metastasis but did not

\section{Biomed Central}


inhibit primary tumor growth [8]. Subsequent studies have demonstrated that BRMS1 dramatically suppresses the metastatic phenotype in vitro in cells from several othertypes of cancer, including melanoma $[9,10]$, ovarian cancer [11], bladder cancer [12] and lung cancer [13]. BRMS1 was also shown to inhibit metastasis in xenograft models of breast cancer [8], melanoma [9] and ovarian carcinoma [11]. However, there are no published reports on in vitro or in vivo BRMS1 expression and function in NPC and no available articles that address a possible relationship between BRMS1 expression and clinical outcomes in NPC.

In this study, we aimed to examine BRMS1 expression and functional status in vitro and in vivo and to explore its clinical significance in clinical NPC specimens. The results may help to understand the mechanisms of metastasis in NPC and provide information for development of personalized therapies for NPC patients with distant metastasis.

\section{Methods}

Study design

Results of qRT-PCR and Western blotting indicated that BRMS1 expression was low in NPC cells. Therefore we created cell lines stably overexpressing BRMS1 or the corresponding empty vector. The effect of BRMS1 on migration and invasion was observed in artificial wound healing assay, transwell invasion array in vitro. A murine model of NPC was constructed to further investigate the metastasis-inhibiting effects of BRMS1 compared with vector ( $n=8$ per group). To determine the clinical significance of BRMS1 in NPC patients, we detected BRMS1 expression by immunohistochemistry in 274 tumor specimens which were randomly divided into a training set $(n=120)$ and a testing set $(n=154)$. The association between BRMS1 expression and patient outcomes was explored in the training set and then validated in the testing set and overall patient population.

\section{Cell lines and cell culture}

The NPC cell lines (SUNE-1, CNE-1, C666-1, CNE-2 and HNE-1) and the immortalized nasopharyngeal epithelial cell line (NP69) were the kind gifts of Professor Zeng Mu-sheng at Sun Yat-sen University Cancer Center (SYSUCC). All NPC cell lines were maintained in RPMI 1640 (Invitrogen, Beijing, China) supplemented with $10 \%$ fetal bovine serum (Gibco, Montevideo, Uruguay), while the NP69 cells were cultured in Keratinocyte-SFM (Invitrogen, Auckland, NZ) supplemented with bovine pituitary extract, as previously described [14]. All the cell lines were incubated at $37^{\circ} \mathrm{C}$ in a $5 \% \mathrm{CO}_{2}$ incubator.

\section{RNA extraction and qRT-PCR}

The total RNA was extracted from the cell lines using TRIzol reagent (Life Technologies, Grand Island, NY).
The first-strand cDNA was synthesized using the M-MLV First-strand Synthesis Kit (Invitrogen, China). The following PCR primers were used for BRMS1 and glyceraldehyde3-phosphate dehydrogenase (GAPDH): BRMS1 forward, 5'-AAGGCACCTCTGGTTTCTGG-3'; BRMS1 reverse, 5'-TGTGAACAGCAGGGTCAAGGT-3'; GAPDH forward, 5'-CTCCTCCTGTTCG ACAGTCAGC-3' and GAPDH reverse, 5'-CCCAATACGACCAAATCCGTT-3'. The quantitative PCR was performed using SYBR Green qPCR SuperMix-UDG reagent (Invitrogen, China) and an ABI PRISM 7900HT sequence detection system (Applied Biosystems, USA). The BRMS1 cycle threshold (Ct) was normalized to the GAPDH internal reference.

\section{Western blotting}

The protein was extracted as previously described [14] , loaded onto 12\% SDS-PAGE gels and transferred to PVDF membranes. The membranes were blocked with a mouse anti-human BRMS1 antibody (1:500; Abnova, Taipei, Taiwan). BRMS1 expression was detected with horseradish peroxidase-conjugated goat anti-mouse antibody (1:10,000, Merck, Darmstadt, Germany) and a Super Signal enhanced chemiluminescence substrate (Pierce, Rockford, IL, USA). A rabbit anti-human $\alpha$ tubulin antibody (1:1000, CST, USA) was used to confirm equal loading.

\section{Establishing NPC cells that stably expressed BRMS1}

Following the manufacturer's instructions, the CNE-2 and SUNE-1 cell lines were stably transfected using a Lenti-Pac $^{\mathrm{T} \mathrm{M}}$ HIV Expression Packaging Kit (GeneCopoeia, Rockville, MD, USA) and a plasmid encoding BRMS1 or a control vector plasmid. Briefly, EndoFectin Lenti reagent was used to transfect the parental CNE-2 and SUNE-1 cells with $2.5 \mu \mathrm{g}$ of either a lentiviral BRMS1 ORF plasmid (EX-V1241-Lv105, GeneCopoeia) or a control vector plasmid (EX-NEG-Lv105, GeneCopoeia). The cells were allowed to grow under puromycin $(0.5 \mu \mathrm{g} / \mathrm{ml})$ selection for 10 days. Western blotting and qRT-PCR were used to analyze the BRMS1 expression. The cells overexpressing BRMS1 were renamed CNE-2B and SUNE-1B, and the vector control cells were renamed CNE-2V and SUNE-1V.

\section{Wound healing assay}

The CNE-2B/V and SUNE-1B/V cells were seeded in 6-well cell culture plates. When the cell confluence reached approximately 90\%, the cells were serumstarved for $24 \mathrm{~h}$, and wounds were then created by scraping the cell monolayer with a $200-\mu l$ pipette tip. The cells were then rinsed with serum-free medium to remove floating cells and debris. The culture plates were incubated at $37^{\circ} \mathrm{C}$. The width of the wounds was measured at various times. Representative wounds were 
photographed under a phase-contrast inverted microscope ( $4 \times$ objective, Leica, Wetzlar, Germany). The experiment was repeated three times.

\section{Transwell invasion assays}

The log phase CNE-2B/V and SUNE-1B/V cells were trypsinized and suspended in single cell solutions. A total of $1 \times 10^{5}$ cells in $200 \mu \mathrm{l}$ serum-free RPMI 1640 medium were seeded on $8-\mu \mathrm{m}$-pore polycarbonate membrane chambers in Transwell plates (Corning, Corning, NY, USA) that were coated Matrigel (BD Biosciences, San Jose, CA), and $600 \mu \mathrm{l}$ of RPMI 1640 containing 20\% FBS was added to the lower chamber. After incubation for 18 hours at $37^{\circ} \mathrm{C}$ in a $5 \% \mathrm{CO}_{2}$ incubator, the cells on the top insert surface were removed by wiping with a cotton swab. The cells that had invaded to the bottom surface of the insert were fixed with a 3:1 mixture of methanol and acetic acid for 10 minutes, stained in $0.5 \%$ crystal violet for 30 minutes, rinsed in PBS and then subjected to microscopic inspection $(200 \times)$. The numbers of invading cells were obtained by counting the number of cells in five random microscopic fields per membrane. The experiment was repeated three times.

\section{In vivo lung metastasis model}

Male BALB/c nude mice between 5 and 6 weeks old were purchased from the Hunan Slac Jingda Laboratory Animal Co., Ltd. (Changsha, Hunan province, China) and were in quarantined for a week before treatment. Animals were provided with standard laboratory chow and tap water ad libitum under special pathogen free (SPF) conditions. All the animal studies were conducted in accordance with "Animal Research: Reporting In Vivo Experiments" (ARRIVE) guidelines and the guidelines of Institutional Animal Care and Use Committee at SYSUCC. All mice were treated humanely throughout the experimental period.

To assay for lung metastases, $1 \times 10^{6} \mathrm{SUNE}-1 \mathrm{~B} / \mathrm{V}$ cells in $200 \mu \mathrm{l}$ PBS were injected into the lateral tail veins of the mice ( $n=8$ per group). Nine weeks later, the mice were necropsied after anesthesia. The lungs of mice were fixed in $3.7 \%$ formaldehyde, $5 \%$ glacial acetic acid, and $72 \%$ ethanol for at least one day before proceeding to paraffin embedding. Serial $5-\mu \mathrm{m}$ sections were cut, and one of every ten slides was stained with H\&E for histopathological examination.

\section{Tissue specimens, patient information and follow-up}

274 biopsy-proven and non-distant-metastasis paraffinembedded NPC samples and 8 noncancerous nasopharyngeal (NNP) tissues were collected at SYSUCC between April 2003 and December 2006. None of the NPC patients received any therapies before biopsies. Prior informed consents from the patients and approval from the medical ethics committee of SYSUCC were obtained. The patients' clinical information is summarized in Table 1. The clinical staging of all the NPC patients was re-performed according to the $7^{\text {th }}$ International Union Against Cancer (UICC)/American Joint Committee on Cancer (AJCC) system [15]. The 274 NPC FFPE specimens were randomly divided into a training set and a testing set using a random number table generated by SPSS 16.0 software (SPSS, Chicago, IL, USA).

After completing their therapy, the patients returned for follow-up appointments every 3 months for the first 2 years and every 6 months thereafter. The last followup date was April 30, 2011, and the median follow-up period was 61.9 months (range, 3.1-85.4 months). All the events were measured from the date of diagnosis. The following end points were assessed (as the time to the defining event): distant metastasis-free survival (DMFS, with "distant" defined as metastasis to other organs or tissues) and overall survival (OS, with "overall" defined as death due to any cause).

\section{Immunohistochemistry}

Immunohistochemical staining was performed similarly to previous report [14]. The Mouse anti-BRMS1 antibody (1:400; Abnova, Taiwan) and biotinylated antimouse secondary antibody (zsbio, Beijing, China) were used. The degree of immunostaining was reviewed and scored independently by two pathologists. The staining intensity was scored as 0 (negative), 1 (weak), 2 (medium), and 3 (strong). Extent of staining was scored as 0 (0\%), 1 (1\% to $25 \%), 2$ (26\% to $50 \%), 3$ (51\% to $75 \%$ ), and 4 (76 to $100 \%$ ) according to the percentages of positive tumor cells in the tumor area. The final staining score was the sum of the intensity and extent score [16].

\section{Statistical analysis}

The data were expressed as the mean \pm SD. An independent-sample $\mathrm{T}$ test was used to test for significant differences between continuous variables. The distributions of the NPC patients' clinical parameters were compared between the high and low BRMS1 expression groups using the chi-square or Fisher's exact tests. Kaplan-Meier survival analysis was used to compare the patient survival times. The log-rank test was used to evaluate the differences in survival probabilities between the groups. A Cox proportional hazards regression analysis with backward stepwise selection was used to explore the independent predictive factors for DMFS and OS. All the quoted $p$ values are two-sided, and $P<0.05$ was considered to be statistically significant. The statistical analyses were performed using SPSS 16.0 (SPSS, Chicago, IL). 


\begin{tabular}{|c|c|c|c|c|c|c|c|c|c|}
\hline \multirow[t]{2}{*}{ Characteristic } & \multicolumn{3}{|c|}{ Training set $(n=120)$} & \multicolumn{3}{|c|}{ Testing set $(n=154)$} & \multicolumn{3}{|c|}{ Overall patients $(n=274)$} \\
\hline & $\begin{array}{l}\text { low } \\
\text { expression } \\
\text { no. (\%) }\end{array}$ & $\begin{array}{l}\text { high } \\
\text { expression } \\
\text { no. (\%) }\end{array}$ & $P^{*}$ & $\begin{array}{l}\text { low } \\
\text { expression } \\
\text { no. (\%) }\end{array}$ & $\begin{array}{l}\text { high } \\
\text { expression } \\
\text { no. (\%) }\end{array}$ & $P^{*}$ & $\begin{array}{l}\text { low } \\
\text { expression } \\
\text { no. (\%) }\end{array}$ & $\begin{array}{l}\text { high } \\
\text { expression } \\
\text { no. (\%) }\end{array}$ & $P^{*}$ \\
\hline \multicolumn{10}{|l|}{ Gender } \\
\hline Male & $28(66.7)$ & $60(76.9)$ & 0.226 & $44(77.2)$ & $74(76.3)$ & 0.898 & $72(72.7)$ & $134(76.6)$ & 0.286 \\
\hline Female & $14(33.3)$ & $18(23.1)$ & & $13(22.8)$ & $23(23.7)$ & & $27(27.3)$ & $41(23.4)$ & \\
\hline \multicolumn{10}{|l|}{ Age (years) } \\
\hline$\leq 46$ & $20(47.6)$ & $37(47.4)$ & 0.985 & $31(54.4)$ & $49(50.5)$ & 0.643 & $51(51.5)$ & $86(49.1)$ & 0.401 \\
\hline$>46$ & $22(52.4)$ & $41(52.6)$ & & $26(45.6)$ & $48(49.5)$ & & $48(49.5)$ & $89(50.9)$ & \\
\hline \multicolumn{10}{|l|}{ WHO type } \\
\hline Type III & $40(95.2)$ & $74(94.9)$ & 1.000 & $56(98.2)$ & $94(96.9)$ & 1.000 & $96(97.0)$ & $168(96.0)$ & 0.482 \\
\hline Other type & $2(4.8)$ & $4(5.1)$ & & $1(1.8)$ & $3(3.1)$ & & $3(3.0)$ & $7(4.0)$ & \\
\hline \multicolumn{10}{|l|}{$V C A-\lg A$} \\
\hline$\geq 1: 320$ & $23(54.8)$ & $41(52.6)$ & 0.818 & $28(49.1)$ & $48(49.5)$ & 0.965 & $51(51.5)$ & $89(50.9)$ & 0.917 \\
\hline$<1: 320$ & $19(45.2)$ & $37(47.4)$ & & 29(50.9) & $49(50.5)$ & & $48(48.5)$ & $86(49.1)$ & \\
\hline \multicolumn{10}{|l|}{$E A-\lg A$} \\
\hline$\geq 1: 20$ & $20(47.6)$ & $43(55.1)$ & 0.432 & $33(57.9)$ & $50(51.5)$ & 0.445 & $53(53.5)$ & 93(53.1) & 0.950 \\
\hline$<1: 20$ & $22(52.4)$ & $35(44.9)$ & & $24(42.1)$ & $47(48.5)$ & & $46(46.7)$ & $82(46.9)$ & \\
\hline \multicolumn{10}{|l|}{ AER } \\
\hline$\geq 63 \%$ & $8(19.0)$ & $23(29.5)$ & 0.213 & $17(29.8)$ & $26(26.8)$ & 0.687 & $25(25.3)$ & $49(28.0)$ & 0.623 \\
\hline$<63 \%$ & $34(81.0)$ & $55(70.5)$ & & $40(70.2)$ & $71(73.2)$ & & $74(74.7)$ & $126(72.0)$ & \\
\hline \multicolumn{10}{|l|}{ UICC $7^{\text {th }} T$ stage } \\
\hline $\mathrm{T} 1$ & $5(11.9)$ & $16(20.5)$ & 0.470 & $11(19.3)$ & 18 (18.6) & 0.996 & $16(16.2)$ & $34(19.4)$ & 0.853 \\
\hline $\mathrm{T} 2$ & $17(40.5)$ & $22(28.2)$ & & 18 (31.6) & $32(33.0)$ & & $35(35.4)$ & $54(30.9)$ & \\
\hline $\mathrm{T} 3$ & $9(21.4)$ & $19(24.4)$ & & $16(28.1)$ & $26(26.8)$ & & $25(25.3)$ & $45(25.5)$ & \\
\hline $\mathrm{T} 4$ & $11(26.2)$ & $21(26.9)$ & & $12(21.1)$ & 21 (21.6) & & $23(23.2)$ & $42(23.7)$ & \\
\hline \multicolumn{10}{|l|}{ UICC $7^{\text {th }} N$ stage } \\
\hline No & $4(9.5)$ & $14(17.9)$ & 0.644 & $8(14.0)$ & $14(14.4)$ & 0.837 & $12(12.1)$ & $28(16.0)$ & 0.732 \\
\hline N1 & $22(52.4)$ & $36(46.2)$ & & $24(42.1)$ & $47(48.5)$ & & $46(46.5)$ & $83(47.4)$ & \\
\hline N2 & $10(23.8)$ & $16(20.5)$ & & $15(26.3)$ & $20(20.6)$ & & $25(25.3)$ & $36(20.6)$ & \\
\hline N3 & $6(14.3)$ & $12(15.4)$ & & $10(17.5)$ & $16(16.5)$ & & $16(16.2)$ & $28(16.0)$ & \\
\hline
\end{tabular}


Table 1 The correlations between BRMS1 expression and the clinicopathological characteristics of nasopharyngeal carcinoma (Continued)

\begin{tabular}{|c|c|c|c|c|c|c|c|c|c|}
\hline No & $4(9.5)$ & $14(17.9)$ & 0.307 & $8(14.0)$ & $14(14.4)$ & 0.806 & $12(12.1)$ & $28(16.0)$ & 0.382 \\
\hline Yes & $38(90.5)$ & $64(82.1)$ & & $49(86.0)$ & $83(85.6)$ & & $87(87.9)$ & $147(84.0)$ & \\
\hline \multicolumn{10}{|c|}{ UICC $7^{\text {th }}$ stage } \\
\hline 1 & $0(0.0)$ & $4(5.1)$ & 0.513 & $1(1.8)$ & $3(3.1)$ & 0.949 & $8(2.9)$ & $8(2.9)$ & 0.538 \\
\hline$\|$ & $13(31.0)$ & $22(28.2)$ & & $17(29.8)$ & $27(27.8)$ & & 79 (28.8) & 79 (28.8) & \\
\hline III & $13(31.0)$ & $22(28.2)$ & & 19 (33.3) & $31(32.0)$ & & 85 (31.0) & $85(31.0)$ & \\
\hline IV & $16(38.0)$ & $30(38.5)$ & & $20(35.1)$ & $36(37.1)$ & & $102(37.2)$ & $102(37.2)$ & \\
\hline \multicolumn{10}{|c|}{ Distant metastasis ${ }^{\#}$} \\
\hline No & $24(57.1)$ & $67(85.9)$ & 0.000 & $35(61.4)$ & 83 (85.6) & 0.000 & 59 (59.6) & $150(85.7)$ & 0.000 \\
\hline Yes & $18(42.8)$ & $11(14.1)$ & & 22 (38.6) & $14(14.4)$ & & $40(40.4)$ & 25 (14.3) & \\
\hline
\end{tabular}

* $p$ value was calculated using the chi-squared or Fisher's exact tests. ": The presence of distant metastasis on or before the last follow-up (April 30, 2011). 


\section{Results}

\section{BRMS1 expression is decreased in NPC cells}

Since BRMS1 is a metastasis suppressor in many types of cancer, we hypothesize that NPC progression, especially NPC metastasis, may be related to BRMS1 levels. The results of quantitative reverse transcription-polymerase chain reaction (qRT-PCR, Figure 1A) and western blotting (Figure 1B) indicated that both BRMS1 mRNA and protein were markedly decreased in the examined NPC cell lines compared to the NP69, suggesting that low BRMS1 expression may be involved in NPC progression.

\section{BRMS1 inhibits migration and invasion in vitro}

To confirm that low BRMS1 expression may cause NPC cell metastasis, we conducted an in vitro cell migration and invasion assay using the CNE-2B/V and SUNE-1B/V cell lines that stably expressed BRMS1 or an empty vector. The qRT-PCR and western blot analyses confirmed that BRMS1 mRNA and protein expression were greater in the CNE-2B and SUNE-1B cells than in the corresponding CNE-2V and SUNE-1V control cells (data not shown). The effect of BRMS1 on NPC cell migration was then examined in the wound healing and migration assays. As shown in Figure 2A, the wounded BRMS1-expressing cells (CNE2B and SUNE-1B) traveled a significantly shorter distance after a $24 \mathrm{~h}$ incubation period than did the corresponding vector control cells. In addition, a Transwell invasion assay was conducted to explore the effects of BRMS1 on NPC cell invasion. The results demonstrated that the invasive abilities of the CNE-2B and SUNE-1B cells were significantly lower than those of the corresponding vector cells, indicating massive transmembrane invasion $(P<0.01$ respectively, Figure 2B). These results were further quantified by calculating the number of invading cells per field of high magnification.

\section{BRMS1 inhibits pulmonary metastasis in vivo}

To define the function of BRMS1, a murine xenograft model of distant NPC metastasis was constructed. Upper-left of Figure $2 \mathrm{C}$ showed that there were few metastatic nodules on the surface of excised lungs in the SUNE-1B group, whereas there were many in the control cohort (upper-middle of Figure $-2 \mathrm{C}$ ). This macroscopic observation is quantified in the associated bar graphs, demonstrating a significant decrease in the number of metastatic nodules in the presence of BRMS1 ( $p<0.01$, upper-right of Figure -2C).

In addition, lung sections from each group were stained with $H \& E$ to show the degree of metastasis. The results of the hematoxylin and eosin $(\mathrm{H} \& \mathrm{E})$ staining indicated significantly fewer lung metastasis nodules in the SUNE-1B group than in the SUNE-1V group $(4.9 \pm 3.4$ vs. $20.8 \pm 3.4$, $p<0.01$, lower of Figure $2 \mathrm{C}$ ). These results suggested that BRMS1 expression significantly inhibited xenograft cell invasion in the surrounding tissue. These results above

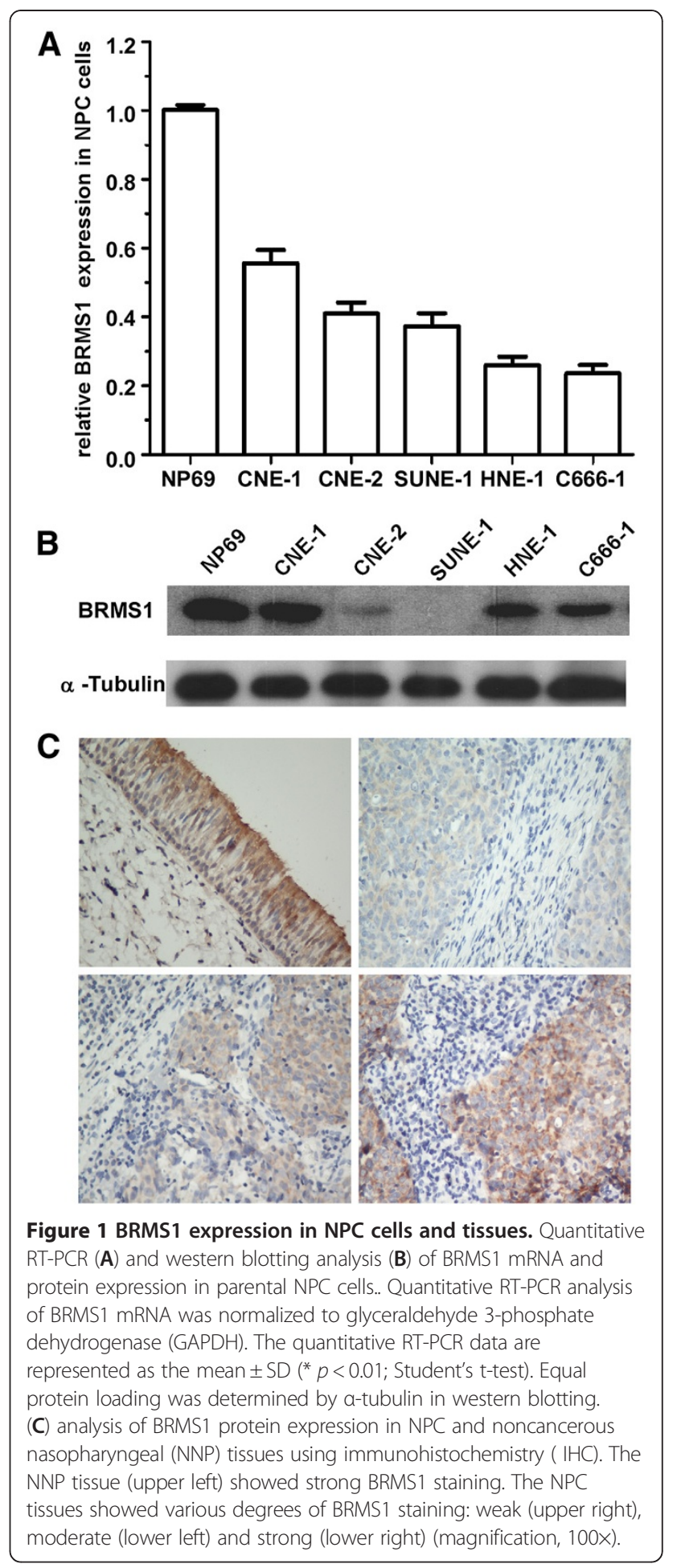

indicated the metastasis-inhibiting role of BRMS1 in this xenograft model.

\section{BRMS1 protein expression is decreased in NPC tissues}

To explore the association between BRMS1 expression and clinical outcome in NPC patients, immunohistochemistry 


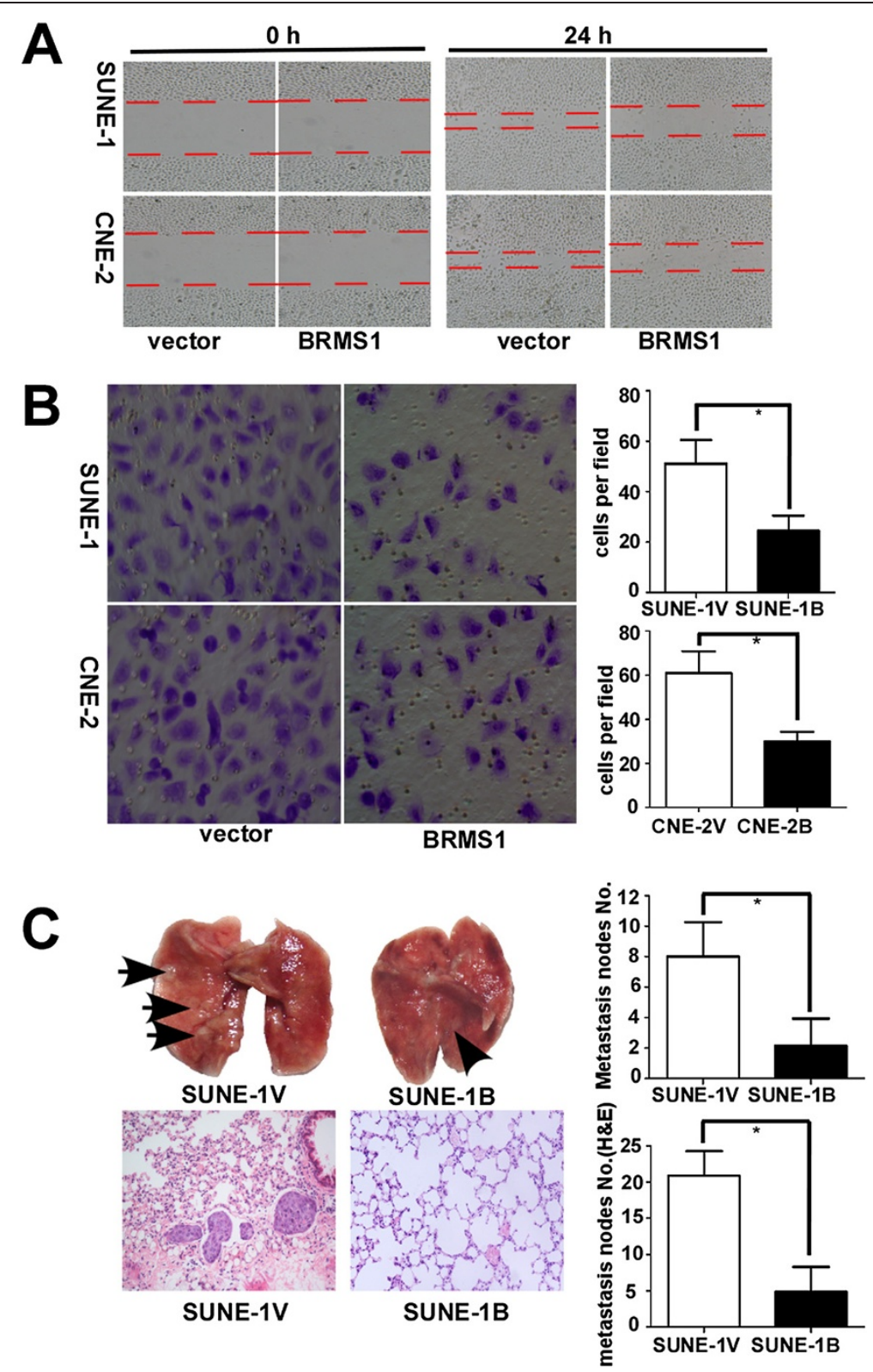

Figure 2 BRMS1 inhibits NPC cell migration and invasion in vitro and suppresses lung metastasis in vivo. (A) The effect of BRMS1 overexpression on cell migration in the wound healing assay. The right panels show a slower wound healing response in the BRMS1overexpressing cells than in the vector control cells $24 \mathrm{~h}$ after scratching (magnification, 40x). (B) The effect of BRMS1 overexpression on cell invasion in the Transwell migration assay. The left panels are representative photomicrographs (magnification, 100x), while the right panels are the numbers of trans-membrane cells per field (magnification, 100X) counted in five random fields for each of the BRMS1-overexpressing and control groups in triplicate parallel experiments. (* $p<0.01$; Student's $t$-test). (C) BRMS1 overexpression inhibits metastasis in murine NPC xenografts. Mouse tumor xenografts were created ( $n=8$ per group). Representative macroscopic photographs of pulmonary metastases; the arrowheads indicate the metastatic nodules on the surface of the lungs (upper left in C). The average numbers of metastases in each group are shown in parallel on the right. Lung sections from each group were stained with hematoxylin and eosin (H\&E) to quantify the degree of lung metastasis (lower left in C). Histograms depicting the average number of microscopic metastases in each group are shown on the right. (* $p<0.01$; Student's t-test).

staining was performed. Representative photomicrographs depicting the BRMS1 staining are shown in Figure 1C. The BRMS1 protein expression was lower in the NPC tissues than in the NNP tissue controls (Figure 1C).
The relationship between BRMS1 expression and clinicopathological features in NPC patients

A receiver operating characteristic (ROC) curve analysis was used to select the cutoff scores for BRMS1 expression 
in the training set, as previously described [17]. The best cutoff scores of BRMS1, viral capsid antigen immunoglobulin A (VCA-IgA), early antigen immunoglobulin A (EA-IgA) and antienzyme rate (AER) of Epstein-Barr virus (EBV) DNase-specific neutralizing antibody for DMFS were 4, 1:320, 1:20 and 63\% respectively (Table 1, Additional file 1: Figure S1).

The clinicopathological features of these two cohorts and the overall patient set, including patient age and gender, WHO pathology type, VCA, EA, AER, tumor stage, node stage, clinical stage and metastasis, are summarized and stratified according to BRMS1 expression in Table 1. We found that in the training set, more patients developed metastasis in the low BRMS1 expression group than in the high BRMS1 expression group $(42.8 \%$ vs. $14.1 \%, p<0.001)$. Similar results were observed in the testing set $(38.6 \%$ vs. $14.1 \%, p<0.001)$ and overall patient set $(40.4 \%$ vs. $14.3 \%, p<0.001)$. No significant associations were found between BRMS1 expression and any of the other clinicopathological features in any set of patients.

\section{Low BRMS1 expression is associated with poor DMFS and OS in NPC patients}

The median follow-up time for the entire patient set was 61.8 months. The cumulative 5-year survival rate was only $52.53 \%$ (95\% confidence interval (CI), 42.69\% $62.36 \%$ ) in the low BRMS1 expression group, whereas it was $74.28 \%(95 \%$ CI, $65.41 \%-81.87 \%)$ in the high BRMS1 expression group. In the training set the patients with low BRMS1 expression had poorer DMFS (upper of Figure3A) and poorer OS (lower of Figure 3A) than those with high BRMS1 expression (hazard ratio (HR) 3.94, 95\% CI, 1.88-8.26, $p<0.001$ for DMFS; HR, $4.78,95 \%$ CI, 2.26-10.10, $p<0.001$ for OS). These results

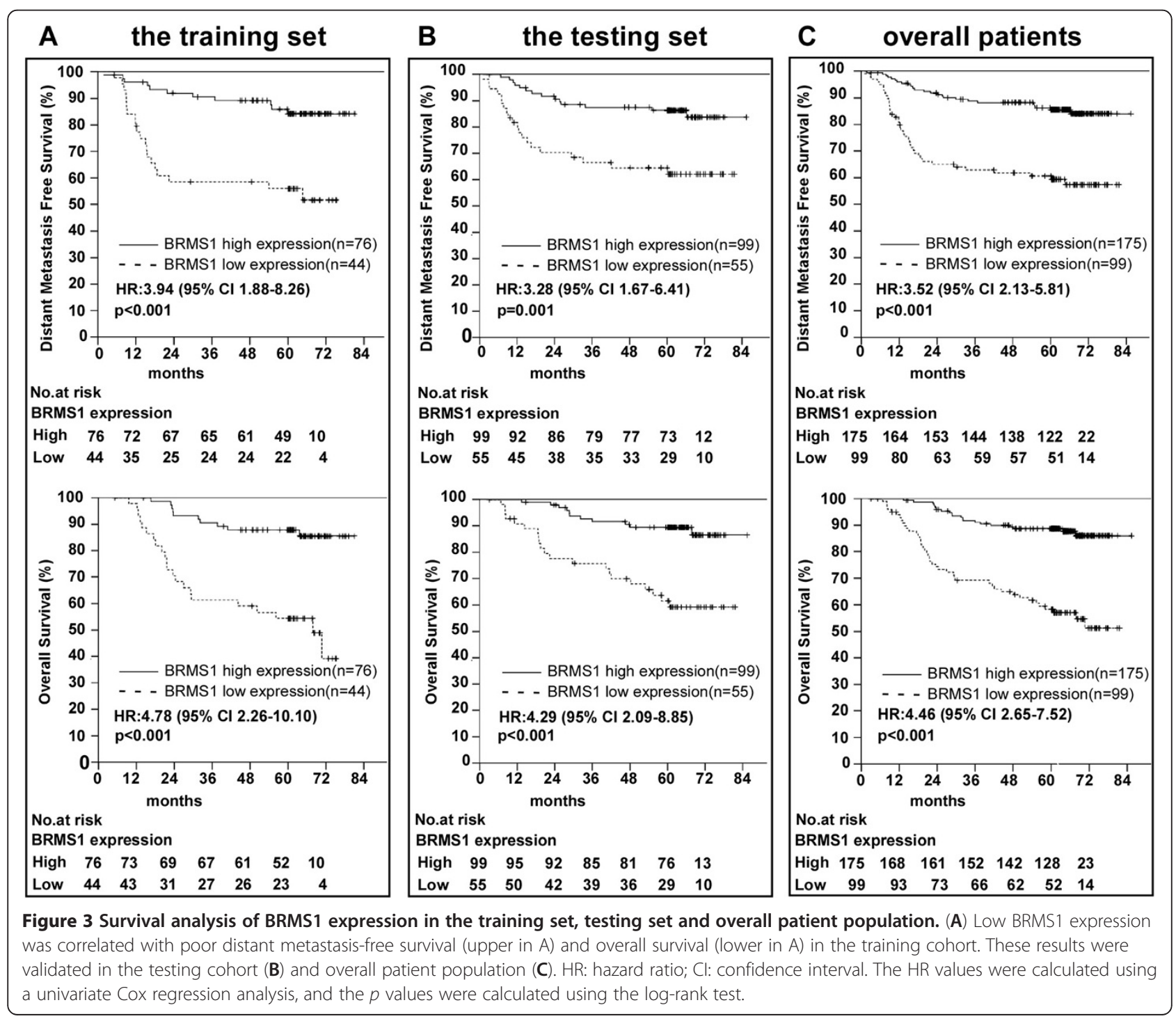


were validated in the testing (Figure $3 \mathrm{~B}$ ) and overall patient sets (Figure 3C).

\section{BRMS1 is an independent prognostic factor for DMFS and $\mathrm{OS}$}

A multivariate Cox regression analysis was performed to explore the effect of various factors on DMFS and OS. Low BRMS1 expression was indeed found to be a significant independent prognostic factor for poor DMFS (HR, 4,54, 95\% CI, $-2.11-9.76, p<0.001$, Table 2) and OS (HR, 6.56, 95\% CI, $2.97-14.41, p<0.001$, Table 2) in the training set. Similar results were also observed in the testing set (HR, 3.31, 95\% CI: $1.69-6.47, p<0.001$ for DMFS and HR, 4.39, 95\% CI, 2.14- 9.01, $p<0.001$ for OS, Table 2) and overall patient population (HR, 3.59, 95\% CI, 2.17-5.92, $p<0.001$ for DMFS and HR, 4.77, 95\% CI, 2.82-8.06, $p<0.001$ for OS, Table 2). In addition, clinical stage was found to be an independent prognostic factor for DMFS and OS in the training, testing and overall patient sets; AER was only an independent prognosis factor in the overall patient population (Table 2). These results indicated that BRMS1 is an independent prognostic factor for DMFS and OS in NPC patients.

\section{BRMS1 is superior to EBV biomarkers for predicting DMFS} and $O S$

The area under ROC curve (AUROC) of BRMS1 for predicting DMFS is bigger than that of VCA, EA and AER

Table 2 A multivariable Cox regression analysis" of BRMS1 expression and survival in nasopharyngeal carcinoma

\begin{tabular}{|c|c|c|c|c|c|c|}
\hline & \multicolumn{3}{|c|}{ For distant metastasis free survival } & \multicolumn{3}{|c|}{ For overall survival } \\
\hline & HR & $95 \% \mathrm{Cl}$ & $P^{*}$ & HR & $95 \% \mathrm{Cl}$ & $P^{*}$ \\
\hline \multicolumn{7}{|l|}{ Training set } \\
\hline sex (men vs. women) & 0.51 & $0.20-1.27$ & 0.15 & 0.29 & $0.11-0.79$ & 0.11 \\
\hline age $(>46$ vs. $\leq 46)$ & 1.16 & $0.55-2.46$ & 0.54 & 1.11 & $0.53-2.35$ & 0.72 \\
\hline WHO type (type I vs. type II) & 0.89 & $0.20-3.99$ & 0.80 & 1.31 & $0.26-5.02$ & 0.81 \\
\hline TNM stage (III, IV vs. I, II) & 3.91 & $1.35-11.29$ & 0.01 & 2.59 & $0.99-6.83$ & 0.04 \\
\hline BRMS1 (low vs. high) & 4.54 & $2.11-9.76$ & 0.00 & 6.56 & $2.97-14.41$ & 0.00 \\
\hline VCA-lgA ( $\geq 1: 320$ vs. $<1: 320)$ & 0.73 & $0.26-2.03$ & 0.93 & 0.41 & $0.15-1.13$ & 0.21 \\
\hline EA-lgA ( $\geq 1: 20$ vs. $<1: 20)$ & 1.44 & $0.52-4.01$ & 0.76 & 1.80 & $0.66-4.92$ & 0.88 \\
\hline AER $(\geq 63 \%$ vs. $<63 \%)$ & 0.73 & $0.36-1.47$ & 0.35 & 1.51 & $0.74-2.51$ & 0.07 \\
\hline \multicolumn{7}{|l|}{ Testing set } \\
\hline sex (men vs. women) & 0.56 & $0.22-1.38$ & 0.28 & 0.76 & $0.36-1.80$ & 0.62 \\
\hline age (> 46 vs. $\leq 46)$ & 1.25 & $0.65-2.43$ & 0.47 & 1.54 & $0.77-3.10$ & 0.14 \\
\hline WHO type (type I vs. type II) & 1.05 & $0.13-8.39$ & 0.60 & 0.67 & $0.08-5.60$ & 0.42 \\
\hline TNM stage (III, IV vs. I, II) & 3.19 & $1.24-8.21$ & 0.02 & 3.91 & $1.38-11.11$ & 0.01 \\
\hline BRMS1 (low vs. high) & 3.31 & $1.69-6.47$ & 0.00 & 4.39 & $2.14-9.01$ & 0.00 \\
\hline VCA-lgA ( $\geq 1: 320$ vs. $<1: 320)$ & 0.47 & $0.19-1.15$ & 0.97 & 0.44 & $0.18-1.10$ & 0.71 \\
\hline EA-lgA ( $\geq 1: 20$ vs. $<1: 20)$ & 2.78 & $1.12-6.94$ & 0.19 & 2.33 & $0.91-5.98$ & 0.31 \\
\hline AER ( $\geq 63 \%$ vs. $<63 \%)$ & 0.57 & $0.26-1.29$ & 0.32 & 1.03 & $0.49-2.19$ & 0.71 \\
\hline \multicolumn{7}{|l|}{ Overall patient population } \\
\hline sex (men vs. women) & 0.63 & $0.34-1.18$ & 0.18 & 0.57 & $0.30-1.07$ & 0.15 \\
\hline age $(>46$ vs. $\leq 46)$ & 1.18 & $0.72-1.92$ & 0.38 & 1.32 & $0.80-2.18$ & 0.25 \\
\hline WHO type (type I vs. type II) & 0.90 & $0.28-2.91$ & 0.74 & 0.89 & $0.27-2.88$ & 0.57 \\
\hline TNM stage (III, IV vs. I, II) & 3.39 & $1.68-6.86$ & 0.00 & 3.24 & $1.60-6.57$ & 0.00 \\
\hline BRMS1 (low vs. high) & 3.59 & $2.17-5.92$ & 0.00 & 4.77 & $2.82-8.06$ & 0.00 \\
\hline VCA-lgA ( $\geq 1: 320$ vs. $<1: 320)$ & 0.62 & $0.31-1.21$ & 0.84 & 0.53 & $0.27-1.04$ & 0.29 \\
\hline EA-lgA ( $\geq 1: 20$ vs. $<1: 20)$ & 1.91 & $0.95-3.85$ & 0.16 & 1.64 & $0.82-3.29$ & 0.72 \\
\hline AER ( $\geq 63 \%$ vs. $<63 \%)$ & 1.20 & $0.70-2.07$ & 0.36 & 1.69 & $1.00-2.86$ & 0.04 \\
\hline
\end{tabular}

\# The variables were selected using the backward Wald stepwise selection method. ${ }^{*}$ The $p$ values were calculated using an adjusted Cox regression model. The following parameters were included as covariates in each of these models: sex (men vs. women), age ( $>46$ vs. $\leq 46)$, WHO type (type I vs. type II), TNM stage (III and IV vs. I and II), BRMS1 expression (low vs. high) VCA-lgA ( $\geq 1: 320$ vs. $<1: 320)$, EA-lgA ( $\geq 1: 20$ vs. $<1: 20)$ and AER $(\geq 63 \%$ vs. $<63 \%)$. VCA-lgA: viral capsid antigenimmunoglobulin A; EA-lgA: early antigenimmunoglobulin A; AER: antienzyme rate (AER) of Epstein-Barr virus (EBV) DNase-specific neutralizing antibody; HR: hazard ratio; Cl: confidence interval. 
respectively in the training set (Additional file 1: Figure S1). Similar trends were found in the testing set and overall patient population (data not shown). These indicated that BRMS1 had a higher accuracy for predicting DMFS than all of the tested EBV biomarkers. Moreover, BRMS1 demonstrated a higher ability to predict OS than VCA, EA and AER in the three cohorts (data not shown). As described above, multivariate Cox regression analysis indicated that BRMS1 expression was an independent factor for both DMFS and OS in the training set, testing set and overall patient population; however, VCA and EA were not any prognostic value in any group and AER was only an independent factor of OS in the overall patient population. Therefore, BRMS1 is superior to EBV biomarkers for predicting DMFS and OS.

\section{Discussion}

In this study, we found low BRMS1 expression in the NPC cell lines and tissue specimens. Artificial overexpression of BRMS1 in the NPC cell lines suppressed migration and invasion in vitro and inhibited the formation of pulmonary metastases in vivo in the nude mice. Furthermore, low BRMS1 expression was significantly associated with poor DMFS and OS in the NPC patients. These results suggested that low BRMS1 expression may play important roles in NPC metastatic process. To our knowledge, this is the first study to reveal a correlation between BRMS1 expression and clinical metastasis and survival in NPC patients.

BRMS1 has previously been demonstrated to be a metastasis-suppressing gene in breast cancer $[18,19]$, melanoma [10,20], ovarian cancer [11], bladder cancer [12] and lung cancer $[13,21]$. Low BRMS1 expression levels have been detected in cell lines $[8,9,11-13,22]$ and tissue specimens $[11,13,19]$ from various human cancers. Our study found that BRMS1 expression levels were markedly lower in the NPC cell lines and tissues than in the NP69 and NNP tissues, indicating that downregulation of BRMS1 may play an important role in NPC progression.

To explore the effect of BRMS1 on NPC metastasis, we firstly created NPC cell lines that stably expressed BRMS1 or an empty vector. Increased BRMS1 expression was shown to decrease NPC cells migration and invasiveness in vitro compared to the corresponding control vector. To further define the metastasis-suppressing function of BRMS1, a nude mouse model of NPC metastasis was constructed. The macroscopic and microscopic observations of the metastatic mouse lung nodules indicated that BRMS1 significantly suppressed pulmonary metastasis formation in vivo. All of the functional studies above demonstrate the inhibitory effects of BRMS1 on NPC metastasis, which is consistent with reports on its effects in other cancers. In breast cancer, BRMS1 expression was higher in the neo11/435 metastasis-suppressed hybrid cell line than in the MDA-MB-435 parental line, which is a highly metastatic breast cancer cell line in vitro. In vivo, ectopic BRMS1-expressing MDA-MB-435 cells showed significantly decreased incidence and number of lung and regional lymph node metastases when the cells were orthotopically injected [8]. BRMS1 expression has also been shown to be lower in a highly metastatic human bladder carcinoma cell line (T24T) than in the less metastatic T24 parental cell line. In vivo modeling found that mice inoculated with T24T cells had significantly more metastases than mice inoculated with T24 cells [12]. Similar results were obtained for lung cancer [13], ovarian cancer [9] and melanoma [10,20].

In this study, we further investigated the clinical significance of BRMS1 in NPC patients using immunohistochemistry (IHC) to assay BRMS1 expression in 274 NPC tissue specimens. BRMS1 was hypothesized to be associated with lymph node metastasis, which is usually correlated with distant metastasis. In our study, however, BRMS1 expression did not correlate with nodal status (Table 1). This result was consistent with previous breast $[19,23]$ and lung cancer [13] studies and may imply that BRMS1 plays different roles in lymph node and distant metastases. Li et al. demonstrated that BRMS1 inhibits blood vessel formation in nude mice [10] by regulating ING4 [24]. As expected, the patients with low BRMS1 expression experienced more metastasis during the follow-up period compared to those with high BRMS1 expression, suggesting that BRMS1 can suppress cancer metastasis in NPC patients. Our results were consistent with those obtained for other neoplasms, such as breast cancer [25], melanoma [10] and NSCLC [13]. However, there is some disagreement on the role of BRMS1 in cancer. Lombardi et al. found that higher BRMS1 mRNA expression was associated with poor disease-free and overall survival [26], which is inconsistent with the results of other studies.

In our study, a multivariate Cox regression analysis demonstrated that both TNM stage and BRMS1 expression were independent prognostic factors for DMFS and OS in the training set, and this conclusion was validated in the testing and overall patient sets. Currently, the current TNM staging system is useful for predicting NPC outcome [15]. However, patients with identical disease stages who receive similar treatments often display considerable variability in their clinical outcomes, indicating that the TNM stage is still far from a perfect predictor. EBV biomarkers such as VCA, EA and AER have been widely used in the diagnosis of NPC, but are not appropriate in predicting prognosis. In our study, BRMS1 expression was an independent prognostic factor for both DMFS and OS in the training set, testing set and overall patient population, while VCA and EA had 
no prognostic value in any group and AER was only an independent factor of OS in the overall patient population. Therefore, BRMS1 levels used in conjunction with the TNM staging system have the potential to more effectively predict patient metastasis potential and prognosis and could guide the development of more personalized therapies for this disease.

Although the clinical significance of BRMS1 in many cancers has been established, the mechanisms by which BRMS1 expression is decreased in tumors is still not clear. Some studies have shown that the BRMS1 promoter is methylated in many cancers, which might contribute to low BRMS1 expression. Metge et al. discovered a CpG island (-3477 to -2214$)$ in the BRMS1 promoter which is hypermethylated across several breast cancer cell lines [27]. Another study demonstrated BRMS1 promoter methylation of in lung cancer [21]. A recent study showed that phosphorylation of RelA/p65 promotes DNMT-1 recruitment to chromatin following BRMS1 promoter methylation and transcriptional repression [22]. BRMS1 has also been reported to interact with the $\mathrm{mSin} 3$ chromatin remodeling complex and to recruit histone deacetylases to suppress downstream gene expression [28]. It has been shown that BRMS1 physically interacts with the RelA/p65 subunit of NF-kB and inhibits IkBa phosphorylation, thus negatively regulating the NF-kB pathway $[29,30]$. Several metastasis-related genes, such as epidermal growth factor receptor [31] and osteopontin [32], have been reported to be regulated by BRMS1.

\section{Conclusion}

In summary, this is the first study to demonstrate that reduced BRMS1 expression is associated with metastasis and survival in NPC patients. We found that BRMS1 suppressed NPC metastasis in vitro and in vivo. A survival analysis showed that BRMS1 expression was an independent predictive factor for DMFS and OS in NPC patients. And BRMS1 was better than EBV biomarkers in predicting DMFS and OS. The results of this study suggest that BRMS1 is a potential biomarker for metastasis and prognosis in NPC patients and may provide a basis for developing gene therapy to prevent or treat NPC metastasis.

\section{Additional file}

Additional file 1: Figure S1.

\section{Abbreviations}

NPC: Nasopharyngeal carcinoma; BRMS1: Breast cancer metastasis suppressor 1; qRT-PCR: Quantitative reverse transcription-polymerase chain reaction; ROC: Receiver operating characteristic; H\&E: Hematoxylin and eosin; NNP: Noncancerous nasopharyngeal; DMFS: Distant metastasis-free survival; OS: Overall survival.

\section{Competing interests}

The author(s) declare that they have no competing interests.

\section{Authors' contributions}

$\mathrm{RXC}, \mathrm{NL}, \mathrm{BJH}$ and $\mathrm{QMH}$ were involved in the study design, performed the experiments, and drafted the manuscript. WFL, SY, TLL, CM, and JN participated in sample collection. CL and GY performed the statistical analysis. JPY and JZ reviewed the manuscript. JM and HYW conceived the idea for the study, contributed to the overall experiment design and revised the manuscript. All authors read and approved the final manuscript.

\section{Acknowledgments}

We wish to express warm thanks to Professor Mu-sheng Zeng (Sun Yat-sen University Cancer Center) for providing the NP69 and NPC cell lines (SUNE-1, CNE1, C666-1, CNE-2 and HNE1). We thank Professor Dan Xie (Sun Yat-sen University Cancer Center) for review of the manuscript.

This work was supported by grants from the Science Foundation of the Key Hospital Clinical Program of the Ministry of Health P.R. China (no. 2010-178), the Key Scientific and Technological Innovation Program of the Universities of Guangdong Province (no. cxzd1005) and the Guangdong Province Universities and Colleges Pearl River Scholar Funded Scheme (2010).

\section{Author details}

${ }^{1}$ Department of Radiation Oncology, Sun Yat-sen University Cancer Center, Guangzhou, People's Republic of China. ${ }^{2}$ Present address: State Key Laboratory of Oncology in South China, Sun Yat-sen University Cancer Center, 651 Dongfeng Road East, Guangzhou 510060, Peoples Republic of China. ${ }^{3}$ Department of Pathology, Sun Yat-sen University Cancer Center, Guangzhou, Peoples Republic of China. ${ }^{4}$ Department of National Clinical Study Center for Anticancer Drugs, Sun Yat-sen University Cancer Center, Guangzhou, Peoples Republic of China. ${ }^{5}$ Department of Radiation Oncology State Key Laboratory of Oncology in South China, Sun Yat-sen University Cancer Center, 651 Dongfeng Road East, Guangzhou 510060, Peoples Republic of China.

Received: 9 April 2012 Accepted: 14 August 2012 Published: 29 August 2012

\section{References}

1. Aea J: Global cancer statistics. CA Cancer J Clin 2011, 61(2):69-90.

2. Yu MC, Yuan JM: Epidemiology of nasopharyngeal carcinoma. Semin Cancer Biol 2002, 12(6):421-429.

3. Kwong DL, Sham JS, Au GK, Chua DT, Kwong PW, Cheng AC, Wu PM, Law MW, Kwok CC, Yau CC, et al: Concurrent and adjuvant chemotherapy for nasopharyngeal carcinoma: a factorial study. J Clin Oncol 2004, 22(13):2643-2653.

4. Wee J, Tan EH, Tai BC, Wong HB, Leong SS, Tan T, Chua ET, Yang E, Lee KM, Fong KW, et al: Randomized trial of radiotherapy versus concurrent chemoradiotherapy followed by adjuvant chemotherapy in patients with American Joint Committee on Cancer/International Union against cancer stage III and IV nasopharyngeal cancer of the endemic variety. J Clin Oncol 2005, 23(27):6730-6738.

5. Lee N, Xia P, Quivey JM, Sultanem K, Poon I, Akazawa C, Akazawa P, Weinberg V, Fu KK: Intensity-modulated radiotherapy in the treatment of nasopharyngeal carcinoma: an update of the UCSF experience. Int J Radiat Oncol Biol Phys 2002, 53(1):12-22.

6. Lai SZ, Li WF, Chen L, Luo W, Chen YY, Liu LZ, Sun Y, Lin AH, Liu MZ, Ma J: How does intensity-modulated radiotherapy versus conventional twodimensional radiotherapy influence the treatment results in nasopharyngeal carcinoma patients? Int J Radiat Oncol Biol Phys 2011, 80(3):661-668

7. Germanov E, Berman JN, Guernsey DL: Current and future approaches for the therapeutic targeting of metastasis (review). Int J Mol Med 2006, 18(6):1025-1036

8. Seraj MJ, Samant RS, Verderame MF, Welch DR: Functional evidence for a novel human breast carcinoma metastasis suppressor, BRMS1, encoded at chromosome 11q13. Cancer Res 2000, 60(11):2764-2769.

9. Shevde LA, Samant RS, Goldberg SF, Sikaneta T, Alessandrini A, Donahue HJ, Mauger DT, Welch DR: Suppression of human melanoma metastasis by the metastasis suppressor gene, BRMS1. Exp Cell Res 2002, 273(2):229-239.

10. Li J, Cheng Y, Tai D, Martinka M, Welch DR, Li G: Prognostic significance of BRMS1 expression in human melanoma and its role in tumor angiogenesis. Oncogene 2011, 30(8):896-906. 
11. Zhang S, Lin QD, Di W: Suppression of human ovarian carcinoma metastasis by the metastasis-suppressor gene, BRMS1. Int J Gynecol Cancer 2006, 16(2):522-531.

12. Seraj MJ, Harding MA, Gildea JJ, Welch DR, Theodorescu D: The relationship of BRMS1 and RhoGDI2 gene expression to metastatic potential in lineage related human bladder cancer cell lines. Clin Exp Metastasis 2000, 18(6):519-525.

13. Smith PW, Liu Y, Siefert SA, Moskaluk CA, Petroni GR, Jones DR: Breast cancer metastasis suppressor 1 (BRMS1) suppresses metastasis and correlates with improved patient survival in non-small cell lung cancer. Cancer Lett 2009, 276(2):196-203.

14. Liao WT, Song LB, Zhang HZ, Zhang X, Zhang L, Liu WL, Feng Y, Guo BH, Mai HQ, Cao SM, et al: Centromere protein $\mathrm{H}$ is a novel prognostic marker for nasopharyngeal carcinoma progression and overall patient survival. Clin Cancer Res 2007, 13(2 Pt 1):508-514.

15. Edge SB, Compton CC: The American Joint Committee on Cancer: the 7th edition of the AJCC cancer staging manual and the future of TNM. Ann Surg Oncol 2010, 17(6):1471-1474.

16. Soumaoro LT, Uetake $H$, Higuchi $T$, Takagi $Y$, Enomoto $M$, Sugihara $K$ : Cyclooxygenase-2 expression: a significant prognostic indicator for patients with colorectal cancer. Clin Cancer Res 2004, 10(24):8465-8471.

17. Zlobec I, Steele R, Terracciano L, Jass JR, Lugli A: Selecting immunohistochemical cut-off scores for novel biomarkers of progression and survival in colorectal cancer. J Clin Pathol 2007, 60(10):1112-1116.

18. Stark AM, Tongers K, Maass N, Mehdorn HM, Held-Feindt J: Reduced metastasis-suppressor gene mRNA-expression in breast cancer brain metastases. J Cancer Res Clin Oncol 2005, 131(3):191-198.

19. Hicks DG, Yoder BJ, Short S, Tarr S, Prescott N, Crowe JP, Dawson AE, Budd GT, Sizemore S, Cicek M, et al: Loss of breast cancer metastasis suppressor 1 protein expression predicts reduced disease-free survival in subsets of breast cancer patients. Clin Cancer Res 2006, 12(22):6702-6708.

20. Metge BJ, Liu S, Riker Al, Fodstad O, Samant RS, Shevde LA: Elevated osteopontin levels in metastatic melanoma correlate with epigenetic silencing of breast cancer metastasis suppressor 1. Oncology 2010, 78(1):75-86.

21. Nagji AS, Liu Y, Stelow EB, Stukenborg GJ, Jones DR: BRMS1 transcriptional repression correlates with $\mathrm{CpG}$ island methylation and advanced pathological stage in non-small cell lung cancer. J Pathol 2010, 221(2):229-237.

22. Liu Y, Mayo MW, Nagji AS, Smith PW, Ramsey CS, Li D, Jones DR: Phosphorylation of RelA/p65 promotes DNMT-1 recruitment to chromatin and represses transcription of the tumor metastasis suppressor gene BRMS1. Oncogene 2012, 31(9):1145-1154.

23. Kelly LM, Buggy Y, Hill A, O'Donovan N, Duggan C, McDermott EW, O'Higgins NJ, Young L, Duffy MJ: Expression of the breast cancer metastasis suppressor gene, BRMS1, in human breast carcinoma: lack of correlation with metastasis to axillary lymph nodes. Tumour Biol 2005, 26(4):213-216.

24. Li J, Li G: Cell cycle regulator ING4 is a suppressor of melanoma angiogenesis that is regulated by the metastasis suppressor BRMS1. Cancer Res 2010, 70(24):10445-10453.

25. Zhang Z, Yamashita H, Toyama T, Yamamoto $Y$, Kawasoe $T$, I wase $H$ : Reduced expression of the breast cancer metastasis suppressor 1 mRNA is correlated with poor progress in breast cancer. Clin Cancer Res 2006 12(21):6410-6414

26. Lombardi G, Di Cristofano C, Capodanno A, lorio MC, Aretini P, Isola P, Tancredi M, Collecchi P, Naccarato AG, Porta RP, et al: High level of messenger RNA for BRMS1 in primary breast carcinomas is associated with poor prognosis. Int J Cancer 2007, 120(6):1169-1178.

27. Metge BJ, Frost AR, King JA, Dyess DL, Welch DR, Samant RS, Shevde LA: Epigenetic silencing contributes to the loss of BRMS1 expression in breast cancer. Clin Exp Metastasis 2008, 25(7):753-763.

28. Meehan WJ, Samant RS, Hopper JE, Carrozza MJ, Shevde LA, Workman JL, Eckert KA, Verderame MF, Welch DR: Breast cancer metastasis suppressor 1 (BRMS1) forms complexes with retinoblastoma-binding protein 1 (RBP1) and the mSin3 histone deacetylase complex and represses transcription. J Biol Chem 2004, 279(2):1562-1569.

29. Cicek M, Fukuyama R, Welch DR, Sizemore N, Casey G: Breast cancer metastasis suppressor 1 inhibits gene expression by targeting nuclear factor-kappaB activity. Cancer Res 2005, 65(9):3586-3595.
30. Cicek M, Fukuyama R, Cicek MS, Sizemore S, Welch DR, Sizemore N, Casey G: BRMS1 contributes to the negative regulation of uPA gene expression through recruitment of HDAC1 to the NF-kappaB binding site of the uPA promoter. Clin Exp Metastasis 2009, 26(3):229-237.

31. Vaidya KS, Harihar S, Phadke PA, Stafford LJ, Hurst DR, Hicks DG, Casey G, DeWald DB, Welch DR: Breast cancer metastasis suppressor-1 differentially modulates growth factor signaling. J Biol Chem 2008, 283(42):28354-28360.

32. Samant RS, Clark DW, Fillmore RA, Cicek M, Metge BJ, Chandramouli KH, Chambers AF, Casey G, Welch DR, Shevde LA: Breast cancer metastasis suppressor 1 (BRMS1) inhibits osteopontin transcription by abrogating NF-kappaB activation. Mol Cancer 2007, 6:6

doi:10.1186/1471-2407-12-376

Cite this article as: Cui et al:: Low BRMS1 expression promotes nasopharyngeal carcinoma metastasis in vitro and in vivo and is associated with poor patient survival. BMC Cancer 2012 12:376.

\section{Submit your next manuscript to BioMed Central and take full advantage of:}

- Convenient online submission

- Thorough peer review

- No space constraints or color figure charges

- Immediate publication on acceptance

- Inclusion in PubMed, CAS, Scopus and Google Scholar

- Research which is freely available for redistribution

Submit your manuscript at www.biomedcentral.com/submit
C) Biomed Central 